In 1994 Proc. of IEEE Asilomar Conf. on Signals, Systems, and Compu ters

\title{
FAMILIES OF SMITH FORM DECOMPOSITIONS TO SIMPLIFY MULTIDIMENSIONAL FILTER BANK DESIGN
}

\author{
Brian L. Evans, Jürgen Teich, and Ton A. Kalker \\ Dept. of Electrical Engineering and Computer Sciences \\ University of California, Berkeley, CA 94720-1772 USA \\ E-mail: $\{$ ble,teich, kalker $\}$ deecs . berkeley .edu
}

\begin{abstract}
Imposing structure on the Smith form of an (integer) periodicity matrix $N=U \Lambda V$ leads to efficient $m-D$ DFT implementations. For resampling matrices, i.e. non-singular rational matrices, we introduce Smith form decomposition algorithms to generate $\Lambda$ matrices whose diagonal elements exhibit minimum variance and $U$ matrices with minimum norm. Such structure simplifies non-uniform $m$-D filter bank design.
\end{abstract}

\section{Introduction}

In $\mathrm{m}$-D signal processing, a sampling matrix describes the uniform sampling of $\mathrm{m}-\mathrm{D}$ data because its column vectors form the basis vectors of the sampling grid [1]. Once discretized, the sampled m-D data may be resampled onto a different sampling grid. Resampling the sampled signal in the analog domain requires a conversion of the data to analog form and a resampling with a different scheme (which may possibly require additional hardware). Just as in the 1-D case, performing the resampling in the digital domain avoids the introduction of noise from digital-to-analog and analog-todigital conversion and does not need additional hard-

B. L. Evans was supported by the Ptolemy project. J. Teich was supported by a grant from the German government (DFG), and received supplemental support from the Ptolemy project. B. L. Evans can be reached by phone at $+1510643-6686$ and by fax at $+1510642-2739$. The Ptolemy project is supported by the Advanced Research Projects Agency and the U.S. Air Force (under the RASSP program, contract F33615-93-C-1317), Semiconduct or Research Corporation (project 94-DC-008), National Science Foundation (MIP-9201605), Office of Naval Research (via Naval Research Laboratories), the State of California MICRO program, and the following companies: Bell Northern Research, Dolby, Hitachi, Mentor Graphics, Mitsubishi, NEC, Pacific Bell, Philips, Rockwell, Sony, and Synopsys. World Wide Web information about the Ptolemy project is located at URL http://ptolemy . eecs.berkeley .edu.

T. A. Kalker is employed by Philips Research Laboratories, Eindoven, Prof. Holstlaan, 5656 AA, The Netherlands. ware because a digital computer can efficiently perform the resampling.

In the digital domain, resampling from one grid to another is carried out by mapping the grid coordinates by a rational non-singular matrix known as a resampling matrix. Since a rational matrix can be written as a rational number times an integer matrix, resampling matrices include integer non-singular matrices such as periodicity matrices [1,2] and up/downsampling matrices $[2,3]$. When the resampling matrix is diagonal, the resampling grid is rectangular and the resampling operation is separable. In general, however, resampling matrices are not diagonal, and a transformation is required to decouple the $\mathrm{m}-\mathrm{D}$ dependencies. One common transformation is known as the Smith form decomposition [2, 3, 4, 5].

The Smith form decomposes a resampling matrix $S$ into a product of three "simpler" resampling matrices $U \Lambda V$, and each component matrix has the same dimensions as $S$ (e.g., $2 \times 2$ for 2 -D resampling). $U$ and $V$ are regular unimodular integer matrices because their determinant is \pm 1 , and $\Lambda$ is a diagonal integer matrix for which $|\operatorname{det} \Lambda|=|\operatorname{det} \mathcal{S}|$. The resampling grid associated with $S$ is formed by a linear mapping of samples on an integer grid first by $V$, then by $\Lambda$, and finally by $U$. The linearly mapping of samples by a regular unimodular matrix (or its inverse) corresponds to a shuffling or lossless rearrangement of samples. Thus, downsampling by $S$ can be decomposed into a shuffling of input samples by $U$, followed by a separable downsampling by $\Lambda$, and followed by reshuffling of samples by $V$ [4].

One method for designing m-D non-uniform filter banks [6] first decomposes the resampling matrix $R$ associated with each channel into Smith Form $U_{R} \Lambda_{R} V_{R}$. Then, by cascading the analysis/synthesis sections, the down/upsampling associated with $V_{R}$ cancels. To simplify the design of the analysis/synthesis filters, the skewing from the indexing of the data by each $U_{R}$ matrix and the non-uniform separable resampling by each 
$\Lambda_{R}$ matrix should be minimized. This is equivalent to making the columns of $U_{R}$ be as close to orthogonal as possible and making the diagonal elements of each diagonal matrix $\Lambda_{R}$ be as close to being equal as possible. More formally, we want to design $U_{R}$ such that its lower and upper frame bounds, $F_{\min }$ and $F_{\max }$ respectively, are as close to 1 as possible, where $F_{\min }$ and $F_{\text {max }}$ are defined as the minimum and maximum, respectively, of the set [7]

$$
\left\{\left\|U_{R} \mathbf{f}\right\|:\|\boldsymbol{f}\|=1\right\}
$$

This paper introduces a new algorithm to decompose a resampling matrix into a Smith form that meets the above requirements, which we will call the minimized Smith equalized form. Section 2 reviews computing Smith form decompositions and imposing structure on them. Section 3 establishes the minimization problem being solved and gives a simple example of finding Smith equivalent form. Section 4 gives an efficient algorithm to compute the minimized Smith equivalent form and states the relevant theorems underlying the procedure. Section 5 summarizes the results in the paper, which are not confined to any particular number of dimensions.

\section{Smith Form Decompositions}

The Smith form decomposition of a non-singular integer matrix $\mathcal{S}=U \Lambda V$ was first reported by H. J. S. Smith in 1861. An algorithm to find it iteratively multiplies the given integer matrix on the left and right by elementary (regular unimodular) matrices until the matrix is reduced to a diagonal form [8]. The initial step in the decomposition of an $n \times n$ integer matrix $\mathcal{S}$ sets $U=I_{n \times n}, \Lambda=\mathcal{S}$, and $V=I_{n \times n}$. In the first iteration, an element of $\Lambda$ is pivoted to the $(1,1)$ position by multiplying on the left to interchange two rows and on the right to interchange two columns. Each component in the first row and column, except the $(1,1)$ element which is now the pivot, is then reduced modulo the pivot by subtracting a multiple of the pivot. Then, a new pivot is chosen and the process is repeated until all of the components in the first row and column, except for the $(1,1)$ position, are zero. The pivoting is then performed at the $(2,2)$ position and so on until $\Lambda$ is diagonalized. The only degree of freedom in this algorithm is the criteria to choose the pivot at each iteration. In [8], the pivot is chosen to be the element that is smallest absolute value.

The algorithm above assumed that the matrix to be decomposed contains only integer components. Smith forms of rational matrices can be computed by first factoring out $1 / d$ where $d$ is the least common multiple of all of the denominators of the matrix. The resulting integer matrix is then decomposed into its Smith form. Each diagonal element of the $\Lambda$ matrix is divided by $d$ to produce the Smith-McMillan form (the m-D analog of rational sampling rate changers) [2]. Even though $\Lambda$ becomes a non-singular rational matrix, $U$ and $V$ remain square regular unimodular integer matrices.

Smith forms $\mathcal{S}=U \Lambda V$ are, however, not unique. The $n \times n$ matrix $\mathcal{S}$ is being mapped into two full $n \times n$ matrices (such that their determinants are \pm 1 ) and one diagonal $n \times n$ matrix (such that the product of the diagonals equals the determinant of $\mathcal{S}$ in absolute value). The Smith form has many more degrees of freedom than the original matrix.

For example, alternate Smith forms can be generated by any pair of regular unimodular matrices $X$ and $Y$ for which the product $X \Lambda Y$ is a diagonal matrix. Because the inverse of a regular unimodular matrix is regular unimodular and the product of two regular unimodular matrices is regular unimodular,

$$
\begin{aligned}
U \Lambda V & =U\left(X^{-1} X\right) \Lambda\left(Y Y^{-1}\right) V \\
& =\left(U X^{-1}\right)(X \Lambda Y)\left(Y^{-1} V\right) \\
& =\bar{U} \bar{\Lambda} \bar{V}
\end{aligned}
$$

One application of this equation is to map a Smith form into its canonical form [8]. Another important application of this equation is to derive the conditions to pivot factors along the diagonal elements and the matrices to perform the pivoting [5].

The matrix product $X \Lambda Y$ can move an integer factor $i$ from the $l$ th diagonal entry to the $k$ th diagonal entry [5]. $X$ and $Y$ (with $\operatorname{det} X= \pm 1$ and $\operatorname{det} Y= \pm 1$ ) are each an identity matrix except for $2 \times 2$ submatrix, thereby reducing $X \Lambda Y=\bar{\Lambda}$ to

$$
\begin{array}{r}
{\left[\begin{array}{cc}
x_{k k} & x_{k l} \\
x_{l k} & x_{l l}
\end{array}\right]\left[\begin{array}{cc}
\lambda_{k} & 0 \\
0 & i \lambda_{l}
\end{array}\right]\left[\begin{array}{cc}
y_{k k} & y_{k l} \\
y_{l k} & y_{l l}
\end{array}\right]=} \\
\\
{\left[\begin{array}{cc}
i \lambda_{k} & 0 \\
0 & \lambda_{l}
\end{array}\right]}
\end{array}
$$

After multiplying both sides by $X^{-1}$ and dividing both sides by $\gamma=\operatorname{gcd}\left(\lambda_{k}, \lambda_{l}\right)$,

$$
\begin{aligned}
& {\left[\begin{array}{cc}
\hat{\lambda}_{k} & 0 \\
0 & i \hat{\lambda}_{l}
\end{array}\right]\left[\begin{array}{ll}
y_{k k} & y_{k l} \\
y_{l k} & y_{l l}
\end{array}\right]=} \\
& \quad \frac{1}{\operatorname{det} X}\left[\begin{array}{cc}
x_{l l} & -x_{k l} \\
-x_{l k} & x_{k k}
\end{array}\right]\left[\begin{array}{cc}
i \hat{\lambda}_{k} & 0 \\
0 & \hat{\lambda}_{l}
\end{array}\right]
\end{aligned}
$$

where $\hat{\lambda}_{k}=\lambda_{k} / \gamma$ and $\hat{\lambda}_{k}=\lambda_{l} / \gamma$. Without loss of 
generality, $\operatorname{det}(X)$ is set to 1 , and after some algebra,

$$
\begin{array}{ll}
x_{k k}=i y_{l l} & x_{k l}=\mu \hat{\lambda}_{k} \\
x_{l k}=-\nu \hat{\lambda}_{l} & x_{l l} \text { is "free" } \\
y_{k k}=i x_{l l} & y_{k l}=-\mu \hat{\lambda}_{l} \\
y_{l k}=\nu \hat{\lambda}_{k} & y_{l l} \text { is "free" }
\end{array}
$$

where $\mu, \nu, x_{l l}$, and $y_{l l}$ are integers constrained by the fact $X$ and $Y$ are regular unimodular integer matrices. Without loss of generality, $\operatorname{det} Y=\operatorname{det} X=1$ :

$$
\begin{aligned}
\operatorname{det} X=\operatorname{det} Y & =x_{l l} y_{l l} i+\mu \nu \hat{\lambda}_{k} \hat{\lambda}_{l} \\
& =\left(x_{l l} y_{l l}\right) i+(\mu \nu)\left(\hat{\lambda}_{k} \hat{\lambda}_{l}\right) \\
& =\alpha i+\beta q=1
\end{aligned}
$$

The condition $\alpha i+\beta q=1$ is the Bezout identity [8] which has a solution if and only if $i$ and $q=\hat{\lambda}_{k} \hat{\lambda}_{l}$ are relatively prime.

Theorem 1 Given a Smith form decomposition of integer matrix $\mathcal{S}=U \Lambda V$, the regular unimodular matrices $X$ and $Y$ applied to the Smith form decomposition according to the equation (1) and defined by equation (4) can move a factor from one diagonal entry of $\Lambda$ to another if and only if $i$ and $\hat{\lambda}_{k}$ are relatively prime and $i$ and $\hat{\lambda}_{l}$ are relatively prime.

Euclid's algorithm can compute the solution to the Bezout identity efficiently [9]. The Bezout numbers $\alpha$ and $\beta$ are not unique. All solutions to the Bezout identity, $\hat{\alpha}$ and $\hat{\beta}$, can be written in terms of an integral parameter $t$ as $\hat{\alpha}=\alpha+t q$ and $\hat{\beta}=\beta-t i$. We can use this degree of freedom to compute $\alpha$ and $\beta$ that will yield the $U$ with the smallest norm [5].

\section{The Minimization Problem}

For $n \times n$ resampling matrix $\mathcal{S}$ in Smith form $\mathcal{S}=U \Lambda V$, we can redistribute the factors of the diagonal elements of $\Lambda$ more evenly using the elementary matrices discussed in the previous section. As mentioned in the previous section,

$$
|\operatorname{det} \mathcal{S}|=|\operatorname{det} \Lambda|=\prod_{i=1}^{n} \Lambda_{i i}=\prod_{i=1}^{n} \lambda_{i}
$$

where the $\lambda_{i}$ terms are the diagonal values of the diagonal matrix $\Lambda$. To minimize the variance, we minimize the arithmetic mean under a given constant geometric mean $g=|\operatorname{det} \mathcal{S}|^{\frac{1}{n}}$. If the $\lambda_{i}$ terms were allowed to take real values, then we could set the arithmetic mean equal to geometric mean, so each diagonal element would be made equal to the geometric mean.
All of the entries of the above matrices, however, must assume integral values. Rational matrices, as previously mentioned, can be rewritten as a rational number times an integer matrix, and this approach would be applied to the integer matrix. In order to minimize the variance of the diagonal entries of $\Lambda$ in the Smith form $\mathcal{S}=U \Lambda V$, we can formulate the following minimization problem:

$$
\begin{aligned}
\min _{\lambda_{i}^{\prime}} & \sum_{i=1}^{n} \lambda_{i}^{\prime} \\
\text { subject to } & \prod_{i=1}^{n} \lambda_{i}^{\prime}=\prod_{i=1}^{n} \lambda_{i} \\
& \lambda_{i}^{\prime} \in Z \text { for } i=1 \ldots n \\
& \mathcal{S}=U^{\prime} \Lambda^{\prime} V^{\prime}
\end{aligned}
$$

In other words, we are trying to find a new set of diagonal elements $\lambda_{i}^{\prime}$ such that their sum is minimized while their product (i.e., the determinant) remains constant. Even though the cost function is linear in the free variables $\lambda_{i}^{\prime}$, the overall minimization is highly non-linear because of the constraints. The constraints require that any mapping from the original set of $\lambda_{i}$ values to another set of allowable $\lambda_{i}^{\prime}$ values must follow Theorem 1 in order to preserve the Smith form decomposition. However, there are many questions concerning a reduced form. The basic problems to be solved are discussed in the next section.

Example 1 As an example of the resulting minimal variance form, we will begin with the diagonal entries of some $\Lambda$ matrix as $\{1,3,90\}$. The representation of each entry into a product of prime numbers raised to an integer power is:

$$
\begin{aligned}
& \lambda_{1}=1=1^{0} \\
& \lambda_{2}=3=1^{0} 2^{0} 3^{1} \\
& \lambda_{3}=90=1^{0} 2^{1} 3^{2} 5^{1}
\end{aligned}
$$

The geometric mean is $g=3 \times 10^{\frac{1}{3}} \approx 6.4633$, and the initial arithmetic mean is $\frac{94}{3}=31 \frac{1}{3}$. At each step in the rearrangement, a factor from one of the diagonal elements whose value is above the geometric mean should be distributed to a diagonal element whose values is below the geometric mean if the resulting arithmetic mean is smaller. Table 1 shows all of the legal pivots (as defined by Theorem 1) of factors of $\lambda_{3}$ to the other two diagonal elements $\lambda_{1}$ and $\lambda_{2}$ and the impact of pivoting on the arithmetic mean. Assuming that we choose the pivot that gives us the smallest arithmetic mean, we pivot the factor $3^{2}$ from the third element to the first element, thereby producing the new diagonal entries $\{9,3,10\}$. In the next iteration, the smallest arithmetic mean occurs when the factor of 2 from the 


$\begin{array}{cccc}\text { Factor } & \text { Pivot } & \text { New Diagonal } & \text { Arithmetic Mean } \\ 2 & \lambda_{3} \text { to } \lambda_{1} & 2,3,45 & 16 \frac{2}{3} \\ 2 & \lambda_{3} \text { to } \lambda_{2} & 1,6,45 & 17 \frac{1}{3} \\ 3 & \lambda_{3} \text { to } \lambda_{1} & 3,3,30 & 12 \\ 3 & \lambda_{3} \text { to } \lambda_{2} & 1,9,30 & 13 \frac{1}{3} \\ 3^{2} & \lambda_{3} \text { to } \lambda_{1} & 9,3,10 & 7 \frac{1}{3} \\ 5 & \lambda_{3} \text { to } \lambda_{1} & 5,3,18 & 8 \frac{2}{3} \\ 5 & \lambda_{3} \text { to } \lambda_{2} & 1,15,18 & 11 \frac{1}{3}\end{array}$

Table 1: Rearrangement of Factors of the Diagonal Entries $\{1,3,90\}$

third diagonal entry is moved to the second. The final diagonal values are $\{9,6,5\}$, and the associated arithmetic mean is 6.667 which is very close to the geometric mean of 6.463 .

\section{Algorithm}

For an $n \times n$ integer resampling matrix $\mathcal{S}$, this section will present an algorithm to compute the minimal variance Smith form of $\mathcal{S}=U \Lambda V$. First, this section will give the representation of $\Lambda$ used by the algorithm. Using the representation, we prove that the minimal variance Smith form is unique up to a permutation of the diagonal elements of $\Lambda$. Then, we state the algorithm.

Our algorithm relies on a representation of the prime factorization of the diagonal elements of the $n \times n$ diagonal matrix $\Lambda$. The representation takes the form of an $n \times m$ matrix, where $m$ is the number of prime factors $p$ appearing in the diagonal elements of $\Lambda$. The entries of the matrix, say $\boldsymbol{\alpha}$, are the exponents of the prime factors:

$$
\lambda_{i}=\Lambda_{i i}=\prod_{j=1}^{m} p_{j}^{\alpha_{j i}}, \text { for } i=1 \ldots n
$$

In Example 1, the diagonal elements of

$$
\Lambda=\left[\begin{array}{ccc}
1 & 0 & 0 \\
0 & 3 & 0 \\
0 & 0 & 90
\end{array}\right]=\left[\begin{array}{ccc}
1^{0} & 0 & 0 \\
0 & 1^{0} 2^{0} 3^{1} & 0 \\
0 & 0 & 1^{0} 2^{1} 3^{2} 5^{1}
\end{array}\right]
$$

contain the four prime factors $p=\{1,2,3,5\}$, so $m=4$. The diagonal elements of $\Lambda$ written as a matrix $\boldsymbol{\alpha}$ of prime factor powers would be

$$
\begin{aligned}
& \boldsymbol{\alpha}=\left[\begin{array}{llll}
0 & 0 & 0 & 0 \\
0 & 0 & 1 & 0 \\
0 & 1 & 2 & 1
\end{array}\right] \\
& \left(1^{\alpha_{j i}}\right) \quad\left(2^{\alpha_{j i}}\right) \quad\left(3^{\alpha_{j i}}\right) \quad\left(5^{\alpha_{j i}}\right)
\end{aligned}
$$

Notice that the prime factor exponents have been sorted down each column. This is in fact the necessary and sufficient condition for $\Lambda$ to be in canonical form, as more formally stated next.

Let $\Lambda$ and $\Lambda^{\prime}$ be two diagonal matrices. Let $\left\{p_{1}, \cdots, p_{m}\right\}$ be the set of all prime factors occurring in any of the diagonal entries of $\Lambda$ or $\Lambda^{\prime}$. Now define the $n \times m$ matrices $\boldsymbol{\alpha}$ and $\boldsymbol{\beta}$ by

$$
\lambda_{i}=\Lambda_{i i}=\prod_{j=1}^{m} p_{j}^{\alpha_{j i}}, \text { for } i=1 \ldots n
$$

and

$$
\lambda_{i}^{\prime}=\Lambda_{i i}^{\prime}=\prod_{j=1}^{m} p_{j}^{\beta_{j i}}, \text { for } i=1 \ldots n
$$

That is, $\boldsymbol{\alpha}$ and $\boldsymbol{\beta}$ are matrices containing the powers of the prime factors of the diagonal elements of $\Lambda$. Using this form, we can easily verify whether or not $\Lambda$ and $\Lambda^{\prime}$ have the same Smith canonical form.

Lemma 1 The matrices $\Lambda$ and $\Lambda^{\prime}$ have the same Smith canonical form if and only if there exists permutations $\sigma_{j} \in S_{m}$ such that $\beta_{i j}=\alpha_{\sigma_{j}(i) j}$.

So, as a particular application of the lemma above, the Smith canonical form of the matrix $\Lambda$ can be found by sorting the columns of the matrix $\boldsymbol{\alpha}$ in ascending order.

Given a resampling matrix $\mathcal{S}$, we find one of its Smith forms $\mathcal{S}=U \Lambda V$. Next, we remove any negative signs on the diagonal entries of $\Lambda$ by multiplying $\Lambda$ on the right by $D$ and by multiplying $V$ on the left by $D^{-1}=D$, where $D$ is the diagonal matrix $D_{i i}=$ $\operatorname{sgn}\left(\Lambda_{i i}\right)$. By factoring the diagonal elements of the new $\Lambda$, we find the matrix $\boldsymbol{\alpha}$ according to equation (7). Next, we consider all possible sets of permutations $\sigma=\left\{\sigma_{j}\right\}$ and find a set of permutations $\sigma_{0}$ that gives the minimum arithmetic mean:

$$
\min _{\sigma} \sum_{i} \prod_{j} p_{j}^{\alpha_{\sigma_{j}(i) j}}
$$

It is well-known that the set of permutations is generated by the set of two-element permutations. For every such elementary permutation of $\sigma_{0}$, Theorem 1 provides a pair of unimodular matrices $X$ and $Y$ realizing that permutation. That is, permuting $p_{j}^{k}$ and $p_{j}^{l}$ is equivalent to pivoting $p_{j}$ raised to the $(k-l)$ th or $(l-k)$ th power, whichever is positive. Therefore, by decomposing the permutations of $\sigma_{0}$ into elementary permutations, we can constructively find a pair of regular unimodular matrices $X$ and $Y$ such that $\Lambda^{\prime}=X \Lambda Y$ is diagonal, and such that the sum of the diagonal entries of $\Lambda^{\prime}$ is minimal among all diagonal matrices with the same Smith canonical form as $\Lambda$. From Section 2, we can set the free parameters of each $X$ and $Y$ pair so as to minimize the norm of $U$. The final result will be a 
Smith form decomposition in the form we desire: the diagonal elements of $\Lambda$ will be as close to each other as possible, and the columns of $U$ will be as orthogonal as possible. A dual of this algorithm exists to minimize $V$. Figure 1 shows an example of computing the Smith form which simultaneously has minimal variance in $\Lambda$ and minimal norm in either $U$ or $V$.

\section{Conclusion}

This paper presents an efficient algorithm to find the Smith form of an integer resampling matrix $\mathcal{S}=U \Lambda V$ so that the diagonal elements of $\Lambda$ have minimal variance. The algorithm first computes a Smith form so that the diagonal elements of $\Lambda$ are positive and ordered. Then, at each iteration, the algorithm applies a square integer regular unimodular matrix to the left and right of $\Lambda$ and the inverse to $U$ and $V$, respectively, to achieve the minimal variance form. The algorithm takes advantage of the two degrees of freedom in each matrix transformation to minimize $U$ with respect to a given norm measure. The final form imposes structure on the Smith form decomposition to simplify the design of non-uniform m-D filter banks [6]. A preliminary version of these algorithms is available for the Mathematica computer algebra environment [10] as the standalone package LatticeTheory.m on the FTP site gauss . eedsp.gatech. edu (IP \#130.207.226.24) in the directory pub/Mathematica. Another version of the lattice theory package is embedded in the signal processing packages which is available on the same FTP site.

\section{References}

[1] D. E. Dudgeon and R. M. Mersereau, Multidimensional Digital Signal Processing. Englewood Cliffs, NJ: Prentice-Hall, Inc., 1984.

[2] T. Chen and P. P. Vaidyanathan, "The role of integer matrices in multidimensional multirate systems," IEEE Trans. on Signal Processing, vol. 41, pp. 10351047, Mar. 1993.

[3] B. L. Evans, R. H. Bamberger, and J. H. McClellan, "Rules for multidimensional multirate structures," IEEE Trans. on Signal Processing, vol. 42, pp. 762771, Apr. 1994.

[4] E. Viscito and J. Allebach, "Design of perfect reconstruction multidimensional filter banks using cascaded Smith form matrices," in Proc. IEEE Int. Sym. Circuits and Systems, (Espoo, Finland), pp. 831-834, June 1988.

[5] B. L. Evans, T. R. Gardos, and J. H. McClellan, "Imposing structure on Smith form decompositions of ra- tional resampling matrices," IEEE Trans. on Signal Processing, vol. 42, pp. 970-973, Apr. 1994.

[6] T. R. Gardos, K. Nayebi, and R. M. Mersereau, "Analysis and design of multi-dimensional, non-uniform band filter banks," in SPIE Proc. Visual Communications and Image Processing, pp. 49-60, Nov. 1992.

[7] I. Daubechies, Ten Lectures on Wavelets. CBMS-NSF regional conference series in applied mathematics; 61 , SIAM, 1992. ISBN 0-89871-274-2.

[8] A. Kaufmann and A. Henry-Labordère, Integer and Mixed Programming: Theory and Applications. New York: Academic Press, 1977.

[9] J. H. McClellan and C. M. Rader, Number Theory in Digital Signal Processing. Englewood Cliffs, NJ: Prentice-Hall, 1979.

[10] S. Wolfram, Mathematica: A System for Doing Mathematics by Computer. Redwood City, CA: AddisonWesley, 1988.

$$
\mathcal{S}=\left[\begin{array}{ccc}
736 & 3060 & 1016 \\
256 & 864 & 308 \\
424 & 1068 & 428
\end{array}\right]
$$

(a) Original resampling matrix

$$
\begin{aligned}
{\left[\begin{array}{ccc}
14 & 5 & 3 \\
-1 & 11 & 11 \\
-7 & -3 & -2
\end{array}\right] \times } & {\left[\begin{array}{ccc}
20 & 0 & 0 \\
0 & 24 & 0 \\
0 & 0 & 36
\end{array}\right] \times } \\
& {\left[\begin{array}{ccc}
-884 & -2901 & -1045 \\
5271 & 17311 & 6234 \\
-3558 & -11685 & -4208
\end{array}\right] }
\end{aligned}
$$

(b) Smith Equalized Form with Minimized $U$

$$
\begin{aligned}
{\left[\begin{array}{ccc}
-110 & 341 & 227 \\
-113 & 341 & 229 \\
-323 & 969 & 652
\end{array}\right] \times } & {\left[\begin{array}{ccc}
20 & 0 & 0 \\
0 & 24 & 0 \\
0 & 0 & 36
\end{array}\right] \times } \\
& {\left[\begin{array}{ccc}
-4 & 21 & 7 \\
9 & 19 & 6 \\
-10 & -13 & -4
\end{array}\right] }
\end{aligned}
$$

(c) Smith Equalized Form with Minimized $V$

The Smith Form routine returned the diagonal elements of $\{4,12,360\}$. The equalized form is obtained by pivoting a factor of 5 from the third to first and a factor of 2 from the third to second positions.

\section{Figure 1: Examples of Smith Equalized Forms}

